\title{
Simple and reliable method for tumor localization during totally laparoscopic gastrectomy: intraoperative laparoscopic ultrasonography combined with tattooing
}

\author{
Takeru Matsuda $^{1}$ - Takeshi Iwasaki ${ }^{2}$ Kenro Hirata ${ }^{2}$ Daisuke Tsugawa ${ }^{3} \cdot$ \\ Yutaka Sugita $^{1} \cdot$ Sonoko Ishida $^{2} \cdot$ Shingo Kanaji $^{1} \cdot$ Yoshihiro Kakeji $^{1}$
}

Received: 20 April 2016/ Accepted: 8 August 2016/Published online: 18 August 2016

(c) The International Gastric Cancer Association and The Japanese Gastric Cancer Association 2016

\begin{abstract}
We have developed a new method to localize a tumor during totally laparoscopic gastrectomy that uses intraoperative laparoscopic ultrasonography combined with preoperative clipping and tattooing. One or 2 days before the surgery, endoscopic clipping was performed just proximal to the tumor, followed by tattooing with India ink at the clipping site. Examination by intraoperative laparoscopic ultrasonography was performed at the tattooed site to detect the clips. The resection line of the stomach was determined with use of the detected clips as a marker of the proximal margin of the tumor. This method was attempted in 14 patients who underwent totally laparoscopic gastrectomy, and the clips were successfully identified in all patients. The clips were visualized as several layers of a hyperechoic bar, which was termed a "ladder sign." The mean time from insertion of the laparoscopic probe to identification of the clips was $2 \mathrm{~min}$. The ladder sign is an important finding in this method.
\end{abstract}

Electronic supplementary material The online version of this article (doi:10.1007/s10120-016-0635-z) contains supplementary material, which is available to authorized users.

Takeru Matsuda

tmatsuda@med.kobe-u.ac.jp

1 Division of Gastrointestinal Surgery, Department of Surgery, Kobe University Graduate School of Medicine, 7-5-2 Kusunoki-chou, Chuo-ku, Kobe 650-0017, Japan

2 Department of Surgery, National Hospital Organization Kobe Medical Center, Kobe, Japan

3 Division of Hepato-Biliary-Pancreatic Surgery, Department of Surgery, Kobe University Graduate School of Medicine, Kobe, Japan
Keywords Intraoperative laparoscopic ultrasonography · Totally laparoscopic gastrectomy · Tumor localization . Ladder sign

\section{Introduction}

Laparoscopy-assisted distal gastrectomy was first reported in 1994 [1] and has been widely accepted as a standard treatment of early gastric cancer. Recently, the number of totally laparoscopic gastrectomies (TLGs) has been increasing because of the successful introduction of the construction of an intracorporeal anastomosis [2-6]. On the other hand, the establishment of an intraoperative tumor localization technique during TLG remains an important issue. Although several methods have been reported to date [7-10], none are considered consistently reliable.

We developed a simple and reliable method that uses examination by intraoperative laparoscopic ultrasonography (ILUS) combined with preoperative endoscopic clipping and tattooing. Although the method using ILUS was reported in 2005 [7], it seems to have been rarely performed because of the associated difficulty. In this study, we report the usefulness of combining ILUS with preoperative clipping and tattooing. Furthermore, a typical ILUS finding obtained with our method is presented in a video.

\section{Materials and methods}

\section{Patients}

Between July 2014 and December 2015,14 patients with gastric cancer underwent TLG by this method. The 
indication for laparoscopic gastrectomy at our institution was a cT1-2N0M0 gastric cancer.

Gastric resection and the construction of anastomosis was performed intracorporeally in all cases. Either construction of a delta-shaped anastomosis or Roux-en-Y reconstruction was selected, depending on the size of the remnant stomach in laparoscopic distal gastrectomy. Rouxen-Y reconstruction was performed in laparoscopic total gastrectomy. D1+ lymphadenectomy was performed in all cases according to the treatment guidelines of the Japanese Gastric Cancer Association [11].

\section{Localization procedure}

All patients underwent endoscopic clipping and tattooing 1 or 2 days before surgery. Four metal clips were applied in a line on the anterior wall just proximal to the tumor (Fig. 1). When the tumor was located at the posterior wall of the stomach, the clips were applied at the corresponding portion of the anterior wall. In such cases, the clipping site was determined by agreement between an endoscopist and a surgeon to minimize the locational error between the clipping site and the actual tumor location. India ink $(0.1 \mathrm{ml})$ was then injected into the clipping site to facilitate identification of the clipping site in the stomach during the ILUS examination.

At the beginning of the surgery, ILUS examination was performed with a laparoscopic probe (L43K) with a 12-MHz transducer (Hitachi Aloka Medical, Tokyo, Japan). The laparoscopic probe was applied on the tattooed serosal surface of the stomach (Fig. 2a). The clips were visualized typically as several layers of a hyperechoic bar approximately $1 \mathrm{~cm}$ in length (Fig. 2b, Video S1), and it

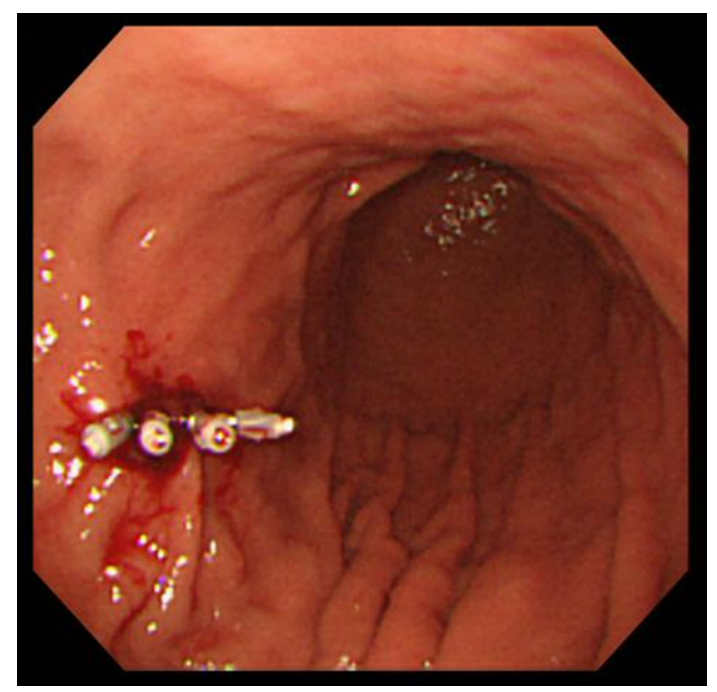

Fig. 1 Preoperative endoscopic clipping and tattooing. Four metal clips were applied in a line on the anterior wall just proximal to the tumor 1 or 2 days before surgery was termed a "ladder sign." A 3-0 monofilament suture was placed on the serosal surface just at the ladder sign. The resection line of the stomach was determined appropriately with the suture as the marker of the proximal margin of the tumor. The stomach was resected intracorporeally with a linear stapler.

\section{Results}

Patient and tumor characteristics are summarized in Table 1. Preoperative clipping and tattooing were performed without complications in all patients. Although the injected ink tended to spread out, the clips were successfully identified by ILUS examination in all cases. The mean time from the insertion of the laparoscopic probe into the peritoneal cavity to the identification of the clips by ILUS examination was $2 \mathrm{~min}$. The clips were typically visualized as the ladder sign (Fig. 2b), which comprises several layers of a strong, hyperechoic bar caused by a clip.

Two of the 14 patients underwent laparoscopic total gastrectomy because of the upper location of the clips; the remaining 12 patients underwent laparoscopic distal gastrectomy. The location of the clips coincided with the marking suture in all cases. Histologic examinations confirmed the resection margins were free of tumor in all cases. The median length of the proximal margin was $27 \mathrm{~mm}$ (range 14-47 $\mathrm{mm}$ ).

\section{Discussion}

With the development and introduction of sophisticated techniques for the construction of an intracorporeal anastomosis during laparoscopic gastrectomy, TLG has been increasingly performed worldwide $[12,13]$. Although it is essential to establish a method for the intraoperative localization of the tumor during TLG, especially for the early tumor located in the middle or upper region of the stomach, a simple and reliable procedure is still unavailable. We developed an effective method that uses ILUS examination combined with preoperative tattooing. A new and reliable finding was also obtained.

So far, in the clinical setting, several techniques for intraoperative identification of the tumor have been reported, such as preoperative or intraoperative tattooing [8, 14], intraoperative endoscopy [15], intraoperative radiography $[9,16]$, and intraoperative ultrasonography [7]. Preoperative tattooing with dye injection is probably the simplest of these, and is often used in colon surgery [17-19]. However, as observed in this study, the injected dye can easily spread across the serosal surface in the case of gastric surgery, which sometimes makes it difficult to 
a
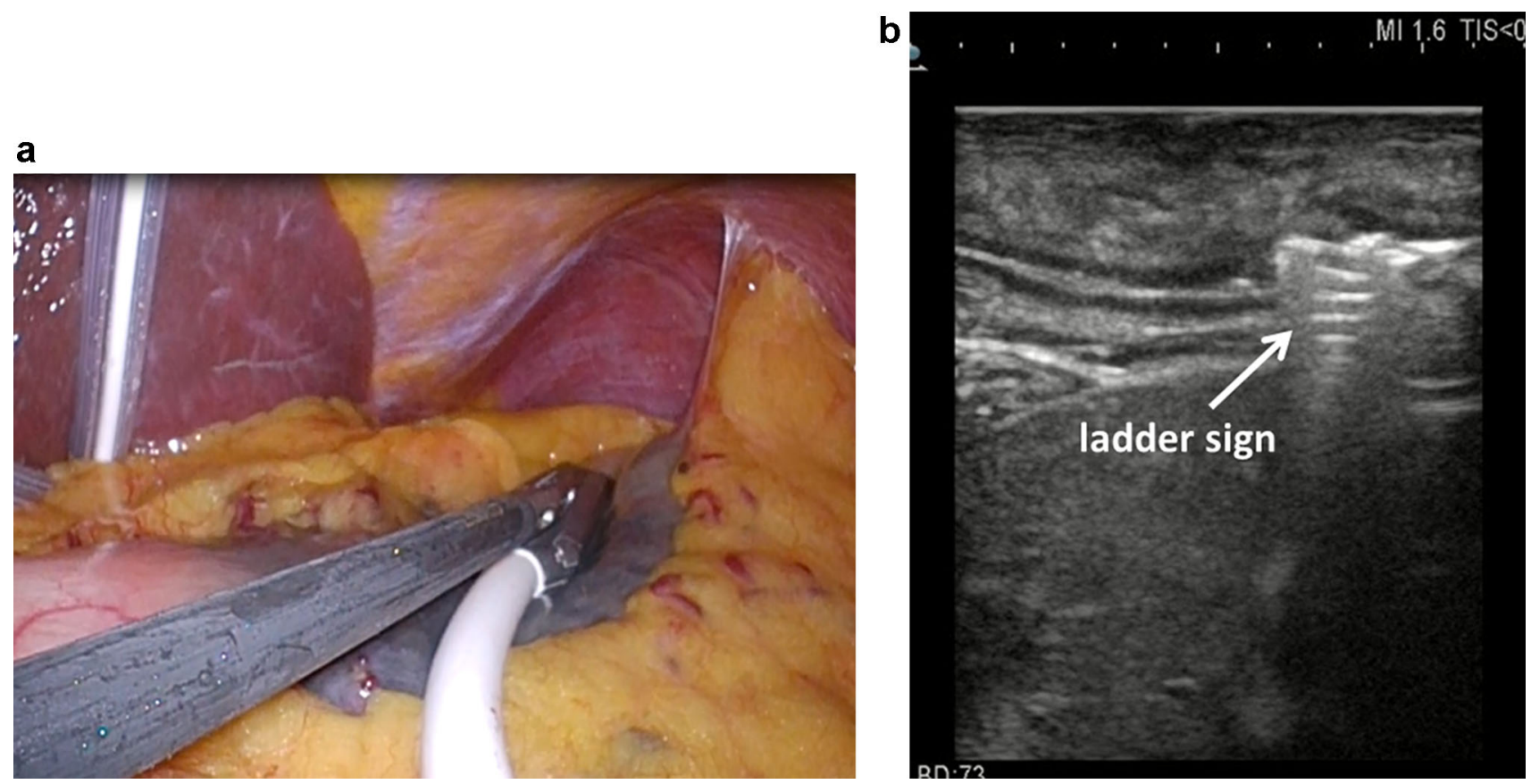

Fig. 2 Localization procedures with examination by intraoperative laparoscopic ultrasonography (ILUS): a ILUS examination was conducted with the laparoscopic probe applied on the tattooed serosal surface of the stomach: b ILUS examination of the clips showing the ladder sign

determine an appropriate resection line. To overcome this disadvantage, Jeong et al. [8] reported blood tattooing instead of dye injection. They reported that the blood-tattooed site measured approximately $3-4 \mathrm{~cm}$ across. Although it is unknown why injected blood stains the serosal surface less diffusely than the conventional dyes, a smaller size of the tattooed site would be ideal because of the importance of precise determination of the extent of stomach resection, not only for oncologic validity but also for secure reconstruction. Nakagawa et al. [14] reported an accurate tattooing method that uses sodium hyaluronate instead of normal saline as the vehicle for patent blue. The tattooed spot seems to be clear and precise when marked with their method. However, because tattoos produced with their mixture become obscured approximately $4 \mathrm{~h}$ after injection, their method requires intraoperative gastroendoscopy, which is still a difficult and complex procedure. Intraoperative radiography seems to be a complicated and time-consuming procedure as well.

Use of ILUS examination was originally reported in 2005 by Hyung et al. [7], who used ILUS without preoperative tattooing. In our experience, it is rather difficult to predict the clipping site precisely without tattooing during ILUS examination. Therefore, endoscopic tattooing with India ink following clipping was used to facilitate easy and rapid identification of the clips by ILUS examination in our method. This tattooing procedure requires additional time and may increase the risk of clinically relevant complications due to tattooing. However, most of the severe complications after tattooing, including intraperitoneal spillage or focal peritonitis, seemed to develop in colorectal surgery because of the much thinner wall of the colon or rectum compared with that of the stomach $[20,21]$. In the present study, preoperative tattooing was successfully performed in all cases, without an adverse effect. We consider the addition of tattooing worthwhile for rapid and secure localization during ILUS examination.

Another important characteristic of our method is the use of high-frequency ultrasonography. We used a laparoscopic probe with a $12-\mathrm{MHz}$ transducer, whereas a 7.5-MHz transducer was used in the previous study [7], probably because a laparoscopic probe for a high-frequency transducer was not available at the time. In recent years, laparoscopic probes have been greatly improved and are considered essential instruments for laparoscopic gastroenterologic surgery, including liver and pancreatic surgery, unlike previously. Therefore, we believe that ILUS examination could be a standard procedure for tumor identification during laparoscopic gastrointestinal surgery.

The ladder sign is a novel finding of the clips identified by ILUS examination. The mechanism of formation of the ladder sign is considered to be multiple reflections of the ultrasound wave. When the ultrasonic probe is applied parallel to a surface that causes a strong reflex, the ultrasound wave is reflected repeatedly between the probe and the reflecting surface, resulting in visualization of strong echo signals in integral multiples of the distance from the probe to the surface [22]. This phenomenon is called 
Table 1 Patient and tumor characteristics $(N=14)$

\begin{tabular}{|c|c|}
\hline Characteristic & Value \\
\hline \multicolumn{2}{|l|}{ Age } \\
\hline Mean (years) & 75 \\
\hline Range (years) & $67-85$ \\
\hline \multicolumn{2}{|l|}{ Sex } \\
\hline Male & $9(64.3 \%)$ \\
\hline Female & $5(35.7 \%)$ \\
\hline \multicolumn{2}{|l|}{ BMI } \\
\hline Mean $\left(\mathrm{kg} / \mathrm{m}^{2}\right)$ & 22 \\
\hline Range $\left(\mathrm{kg} / \mathrm{m}^{2}\right)$ & $18-35$ \\
\hline \multicolumn{2}{|l|}{ ASA classification } \\
\hline 1 & $3(21.4 \%)$ \\
\hline 2 & $10(71.4 \%)$ \\
\hline 3 & $1(7.1 \%)$ \\
\hline \multicolumn{2}{|l|}{ Tumor location } \\
\hline Upper region & $2(14.3 \%)$ \\
\hline Middle region & $10(71.4 \%)$ \\
\hline Lower region & $2(14.3 \%)$ \\
\hline \multicolumn{2}{|c|}{ Tumor location in a cross section } \\
\hline Lesser curvature & $6(42.9 \%)$ \\
\hline Greater curvature & $3(21.4 \%)$ \\
\hline Anterior wall & $3(21.4 \%)$ \\
\hline Posterior wall & $2(14.3 \%)$ \\
\hline \multicolumn{2}{|c|}{ Cellular classification } \\
\hline Intestinal & $8(57.1 \%)$ \\
\hline Diffuse & $6(42.9 \%)$ \\
\hline \multicolumn{2}{|l|}{ pT category ${ }^{\mathrm{a}}$} \\
\hline T1a & $3(21.4 \%)$ \\
\hline $\mathrm{T} 1 \mathrm{~b}$ & $7(50.0 \%)$ \\
\hline $\mathrm{T} 2$ & $2(14.3 \%)$ \\
\hline $\mathrm{T} 3$ & $1(7.1 \%)$ \\
\hline $\mathrm{T} 4$ & $1(7.1 \%)$ \\
\hline \multicolumn{2}{|l|}{ pN category ${ }^{\mathrm{a}}$} \\
\hline No & $9(64.2 \%)$ \\
\hline N1 & $1(7.1 \%)$ \\
\hline $\mathrm{N} 2$ & $3(21.4 \%)$ \\
\hline N3 & $1(7.1 \%)$ \\
\hline \multicolumn{2}{|l|}{ Pathologic stage ${ }^{a}$} \\
\hline I & $9(64.2 \%)$ \\
\hline II & $4(28.6 \%)$ \\
\hline III & $1(7.1 \%)$ \\
\hline
\end{tabular}

ASA American Society of Anesthesiologists, BMI body mass index

a Tumors were classified according to the American Joint Committee on Cancer TNM system

"multiple reflections." Also, when two reflecting surfaces exist close to each other inside an object, the multiple reflections arise between these two surfaces and the strong echo signals appear repeatedly behind the first echo signal. Hence, the ladder sign in our study is probably caused by the multiple reflections between the two lateral surfaces of the clip. The ladder sign can be observed when the axis of the laparoscopic probe corresponds to that of the clip. Therefore, a laparoscopic probe should be applied on the stomach in another direction if the ladder sign cannot be obtained.

One important concern is a locational error of the clips applied to the anterior wall when the tumor is located at the posterior wall. In the present study, a surgeon participated and discussed the clipping site with an endoscopist during endoscopy. However, in the case of the tumor on the posterior wall, we still need to be cautious about determination of the resection line because there was some discrepancy between the actual position of the tumor and the agreed position of the clips. To solve this problem, further studies are definitely required.

In the present study, we demonstrated the usefulness of ILUS examination combined with tattooing to detect the clips applied to the stomach. Although a laparoscopic ultrasonography system is required, this method is simple and reliable. We believe that the clinical value of ILUS examination in laparoscopic gastrointestinal surgery will increase in the future.

\section{Compliance with ethical standards}

Conflict of interest The authors have declared no conflicts of interest.

Ethics statement All procedures in this study were in accordance with the ethical standards of the responsible committee on institutional human experimentation and with the Helsinki Declaration of 1964 and later versions. Informed consent was obtained from all patients for their being included in this study.

\section{References}

1. Kitano S, Iso Y, Moriyama M, Sugimachi K. Laparoscopy-assisted Billroth I gastrectomy. Surg Laparosc Endosc. 1994;4:146-8.

2. Inaba K, Satoh S, Ishida Y, Taniguchi K, Isogaki J, Kanaya S, et al. Overlap method: novel intracorporeal esophagojejunostomy after laparoscopic total gastrectomy. J Am Coll Surg. 2010;211:e25-9.

3. Jeong O, Park YK. Intracorporeal circular stapling esophagojejunostomy using the transorally inserted anvil (OrVil) after laparoscopic total gastrectomy. Surg Endosc. 2009;23:2624-30.

4. Kanaya S, Gomi T, Momoi H, Tamaki N, Isobe H, Katayama T, et al. Delta-shaped anastomosis in totally laparoscopic Billroth I gastrectomy: new technique of intraabdominal gastroduodenostomy. J Am Coll Surg. 2002;195:284-7.

5. Kinoshita T, Oshiro T, Ito K, Shibasaki H, Okazumi S, Katoh R. Intracorporeal circular-stapled esophagojejunostomy using handsewn purse-string suture after laparoscopic total gastrectomy. Surg Endosc. 2010;24:2908-12.

6. Noshiro H, Ohuchida K, Kawamoto M, Ishikawa M, Uchiyama A, Shimizu S, et al. Intraabdominal Roux-en-Y reconstruction 
with a novel stapling technique after laparoscopic distal gastrectomy. Gastric Cancer. 2009;12:164-9.

7. Hyung WJ, Lim JS, Cheong JH, Kim J, Choi SH, Song SY, et al. Intraoperative tumor localization using laparoscopic ultrasonography in laparoscopic-assisted gastrectomy. Surg Endosc. 2005;19:1353-7.

8. Jeong O, Cho SB, Joo YE, Ryu SY, Park YK. Novel technique for intraoperative tumor localization during totally laparoscopic distal gastrectomy: endoscopic autologous blood tattooing. Surg Endosc. 2012;26:1778-83.

9. Kim HI, Hyung WJ, Lee CR, Lim JS, An JY, Cheong JH, et al. Intraoperative portable abdominal radiograph for tumor localization: a simple and accurate method for laparoscopic gastrectomy. Surg Endosc. 2011;25:958-63.

10. Xuan Y, Hur H, Byun CS, Han SU, Cho YK. Efficacy of intraoperative gastroscopy for tumor localization in totally laparoscopic distal gastrectomy for cancer in the middle third of the stomach. Surg Endosc. 2013;27:4364-70.

11. Japanese Gastric Cancer Association. Japanese classification of gastric carcinoma-2nd English edition. Gastric Cancer. 1998:1:10-24.

12. Kinoshita T, Shibasaki H, Oshiro T, Ooshiro M, Okazumi S, Katoh R. Comparison of laparoscopy-assisted and total laparoscopic Billroth-I gastrectomy for gastric cancer: a report of shortterm outcomes. Surg Endosc. 2011;25:1395-401.

13. Song KY, Park CH, Kang HC, Kim JJ, Park SM, Jun KH, et al. Is totally laparoscopic gastrectomy less invasive than laparoscopyassisted gastrectomy?: prospective, multicenter study. J Gastrointest Surg. 2008;12:1015-21.
14. Nakagawa M, Ehara K, Ueno M, Tanaka T, Kaida S, Udagawa H. Accurate, safe, and rapid method of intraoperative tumor identification for totally laparoscopic distal gastrectomy: injection of mixed fluid of sodium hyaluronate and patent blue. Surg Endosc. 2014:28:1371-5.

15. Park DJ, Lee HJ, Kim SG, Jung HC, Song IS, Lee KU, et al. Intraoperative gastroscopy for gastric surgery. Surg Endosc. 2005;19:1358-61.

16. Kim BS, Yook JH, Jung HY. A simplified technique for tumor localization using preoperative endoscopic clipping and radioopaque markers during totally laparoscopic gastrectomy. Am Surg. 2014;80:1266-70.

17. Aboosy N, Mulder CJ, Berends FJ, Meijer JW, Sorge AA. Endoscopic tattoo of the colon might be standardized to locate tumors intraoperatively. Rom J Gastroenterol. 2005;14:245-8.

18. Miyoshi N, Ohue M, Noura S, Yano M, Sasaki Y, Kishi K, et al. Surgical usefulness of indocyanine green as an alternative to India ink for endoscopic marking. Surg Endosc. 2009;23:347-51.

19. Yeung JM, Maxwell-Armstrong C, Acheson AG. Colonic tattooing in laparoscopic surgery-making the mark? Colorectal Dis. 2009;11:527-30.

20. Park SI, Genta RS, Romeo DP, Weesner RE. Colonic abscess and focal peritonitis secondary to india ink tattooing of the colon. Gastrointest Endosc. 1991;37:68-71.

21. Yano H, Okada K, Monden T. Adhesion ileus caused by tattoomarking: unusual complication after laparoscopic surgery for early colorectal cancer. Dis Colon Rectum. 2003;46:987.

22. Yasumoto K. Fundamentals of ultrasound. Nihon Hoshasen Gijutsu Gakkai Zasshi. 2004;60:49-56. 